\title{
Los pacientes terminales fallecen menos en hospitales de agudos aunque aumenta la complejidad de cuidados ofrecidos
}

Fewer terminal patients die in acute care hospitals while complexity of provided care increases

Teno JM, JAMA 2013;309(5):470-477

\section{Objetivo}

Describir los cambios ocurridos en el lugar de fallecimiento, sitio y transición de cuidados recibidos de pacientes con cáncer o enfermedad pulmonar obstructiva crónica, entre 2000 y 2009.

\section{Diseño, lugar y pacientes}

Estudio de cohorte retrospectivo realizado en EE.UU. Se tomó una muestra aleatoria del $20 \%$ de beneficiarios de Medicare, de 66 o más años de edad, con diagnóstico de cáncer, enfermedad pulmonar obstructiva crónica fallecidos en 2000, 2005 y 2009.

\section{Medición de Resultados Principales}

Sitio del fallecimiento, lugar de atención, tasas de transición entre distintos niveles de cuidado, y transiciones "potencialmente onerosas" (p.ej. las reinternaciones en los últimos tres días de vida, o tres o más hospitalizaciones en los últimos 90 días de vida).

Se utilizó una regresión multivariable* para analizar los resultados, después de ajustar por características socio demográficas.

\section{Resultados principales}

Los principales resultados se resumen en la tabla 1. En 2009, el $28,4 \%$ (IC 95\% 27,9 a 28,5\%) del uso de instituciones de cuidados paliativos en el momento de la muerte fue durante tres días o menos. De estos casos el 40,3\% (IC 95\%, 39,7 a 40,8\%) fueron precedidas por la una hospitalización, con una estancia en UCl. Adicionalmente, entre 2000 y 2009 , se duplicó el porcentaje de pacientes que recibieron senvicios de cuidados paliativos en el momento de la muerte.

Tabla 1. Cambios del lugar de atención, muerte, y cuidados recibidos, entre 2000 y 2009

\begin{tabular}{|c|c|c|c|}
\hline & Año 2000 & Año 2005 & Año 2009 \\
\hline $\mathrm{N}^{\circ}$ fallecimientos & 270.202 & 291.819 & 286.282 \\
\hline Muertes en hospitales de agudos, \% (IC 95\%) & $32,6(32,4$ a 32,8$)$ & $26,9(26,7$ a 27,1$)$ & $24,6(24,5$ a 24,8$)$ \\
\hline Utilización de UCI en el último mes de vida, \% (IC 95\%) & $24,3(24,1$ a 24,5$)$ & $26,3(26,1$ a 26,5$)$ & $29,2(29,0$ a 29,3$)$ \\
\hline Muertes en instituciones de cuidados paliativos, \% (IC 95\%) & $21,6(21,4$ a 21,7$)$ & $32,3(32,1$ a 32,5$)$ & $42,2(42,0$ a 42,4$)$ \\
\hline $\begin{array}{l}\text { Transiciones en los últimos } 90 \text { días de vida. Media, [mediana], } \\
\text { (Rango Intercuartilo) }\end{array}$ & $\begin{array}{l}2,1[1,0] \\
(0 \text { a } 3,0)\end{array}$ & $\begin{array}{c}2,8[2,0] \\
(1,0 a 4,0)\end{array}$ & $\begin{array}{c}3,1[2,0] \\
(1,0 \text { a } 5,0)\end{array}$ \\
\hline Transiciones en los últimos tres días de vida, \% (IC 95\%) & $10,3(10,1$ a 10,4$)$ & $12,4(12,3$ a 12,5$)$ & $14,2(14,0$ a 14,3$)$ \\
\hline
\end{tabular}

UCl: unidad de cuidados intensivos. Transición: cambio de institución/proveedor de cuidados.

\section{Conclusiones}

Al comparar los beneficiarios de Medicare fallecidos en 2005 y 2009 contra 2000 se observó que una menor proporción de los mismos falleció en un hospital de agudos, mientras que aumentó la tasa de uti- lización de unidades de cuidados intensivos y la tasa de transiciones durante el último mes de vida.

Fuente de financiamiento: National Institute on Aging y Robert Wood Johnson Foundation.

\section{Comentario}

La elección del sitio de muerte ha sido propuesta como una medida de la calidad de atención al final de la vida, basado en estudios sobre poblaciones norteamericanas que reportan preferencias por morir en el hogar ${ }^{1,2}$. Pero en muchas ocasiones esto no es posible, o no es deseado por el paciente, sus cuidadores o sus médicos. Por otra parte, no puede soslayarse el impacto económico y sanitario del uso intensivo de recursos hospitalarios en situaciones de terminalidad. Las implicancias son múltiples y exceden largamente al problema estrictamente económico (que no es menor). Incluyen cuestiones vinculadas a la autonomía personal, el derecho, la ética profesional, cuestiones de fe y paradigmas culturales relevantes, tanto para los pacientes y sus familias como para el propio equipo de salud, que, en conjunto condicionan la posibilidad de ofrecer a los pacientes los dispositivos de acompañamiento, cuidado y alivio más adecuados para el final de su vida.

En nuestro medio, desconocemos cuales son las preferencias de la población, y dónde efectivamente mueren nuestros pacientes terminales. Aunque los cuidados paliativos se han extendido significativamente en los últimos años, nos falta profundizar en la investigación sistemática sobre nuestra comunidad, la actitud del equipo de salud, y los modelos de gestión más apropiados para las diversas realidades institucionales de nuestro complejo y heterogéneo sistema de salud.

\section{Conclusiones del comentador}

Aunque aumentaron los fallecimientos en el domicilio entre beneficiarios de Medicare de 65 años o más, los resultados obtenidos no son consistentes con la noción de que exista una tendencia temporal hacia cuidados menos agresivos al final de vida en esta población ya que aumentaron la tasa de utilización de UCI y de transiciones en los últimos días de vida. Se debería realizar más investigaciones para establecer si estas tendencias se vinculan con una mayor calidad de vida y si, a su vez, estas son verdaderamente preferidas por los pacientes.

Javier O. Vilosio [ Sección Cuidados Paliativos, Servicio de Clínica Médica del Hospital Italiano de Buenos Aires. javier.vilosio@ hospitalitaliano.org.ar ]

Vilosio JO. Los pacientes terminales fallecen menos en hospitales de agudos aunque aumenta la complejidad de cuidados ofrecidos. Evid Act Pract Ambul Oct-Dic 2013; 16(4):134. Comentado de:Teno JM, Gozalo PL, Bynum JPW, et al. Change in End-of-Life Care for Medicare Beneficiaries. Site of Death, Place of Care, and Health Care Transitions in 2000, 2005, and 2009. JAMA, 2013;309(5): 470-477. PMID 22777380.

Referencias

1. Fried TR y col. Older persons' preferences for site of terminal care. Ann Intern Med. 1999;131(2):109-112.

2. Brazil $\mathrm{K}$ y col. Preferences for place of care and place of death among informal caregivers of the terminally ill. Palliat Med. 2005;19(6):492-499. 